\title{
Almost Optimal Strategies in One Clock Priced Timed Games
}

\author{
Patricia Bouyer ${ }^{1}$, Kim G. Larsen ${ }^{2}$, \\ Nicolas Markey ${ }^{1}$, and Jacob Illum Rasmussen ${ }^{2}$ \\ 1 Lab. Spécification \& Vérification, CNRS \& ENS Cachan, France \\ \{bouyer, markey\}@lsv.ens-cachan.fr \\ 2 Department of Computer Science, Aalborg University, Denmark \\ $\{\mathrm{kgl}, \mathrm{illum}\} @ \mathrm{cs}$. aau.dk
}

\begin{abstract}
We consider timed games extended with cost information, and prove computability of the optimal cost and of $\varepsilon$-optimal memoryless strategies in timed games with one clock. In contrast, this problem has recently been proved undecidable for timed games with three clocks.
\end{abstract}

\section{Introduction}

An interesting direction of real-time model checking that has recently received substantial attention is to extend and re-target timed automata technology towards optimal scheduling and planning $[1,15,9]$. In particular, as part of this effort, the notion of priced timed automata $[6,5]$ has been promoted as a useful extension of the classical model of timed automata [4]. In this extended model each location $q$ is associated with a cost $c_{q}$ giving the cost of a unit of time spent in $q$. Thus, each run of a priced timed automaton has an accumulated cost, based on which a variety of optimization problems may be formulated.

Several of the established results concerning priced timed automata are concerned with reachability questions. In [3] cost-bounded reachability was shown decidable. [6] and [5] independently show computability of the cost-optimal reachability for priced (or weighted) timed automata using different adaptations of the so-called region technique. In $[13,15]$ the notion of priced zone is developed allowing efficient implementation of cost-optimal reachability as witnessed by the competitive tool UPPAAL Cora [16]. Also the problem of computing optimal infinite schedules (in terms of minimal limit-ratios) has been shown computable [8]. Finally cost-optimal reachability has been shown decidable in a setting with multiple cost-variables [14].

In this paper we consider the more challenging problem of the computation of cost-optimal winning strategies for priced timed game automata, i.e. a game where the controller tries to win at minimal cost and opponent tries to maximize the cost. Consider the priced timed game with the single clock $x$ depicted in Fig. 1. Here the (circle) locations $c_{1}$ and $c_{2}$ are controllable whereas (square) locations $u_{1}$ and $u_{2}$ are uncontrollable with cost-rates being $3,4,1$ and 1 , respectively. All four locations have $x \leq 1$ as invariant. Besides transitions between 
these four locations, additional transitions are indicated to (triangle) locations for which the optimal costs of winning (for any value of $x$ ) are assumed to have already been computed (we call those cost functions outside cost functions in the sequel). Obviously, $c_{1}$ and $c_{2}$ have winning strategies for all values of $x$ by uniformly exiting to their respective outside locations (triangle), $c_{1}^{\text {out }}$ and $c_{2}^{\text {out }}$. However, this strategy is, clearly, suboptimal for both locations. Alternatively, consider the superior strategy for $c_{2}$ depicted in Fig. 2. that guarantees cost no larger than depicted in the corresponding cost function. Then it can be shown that this strategy guarantees the optimal cost.

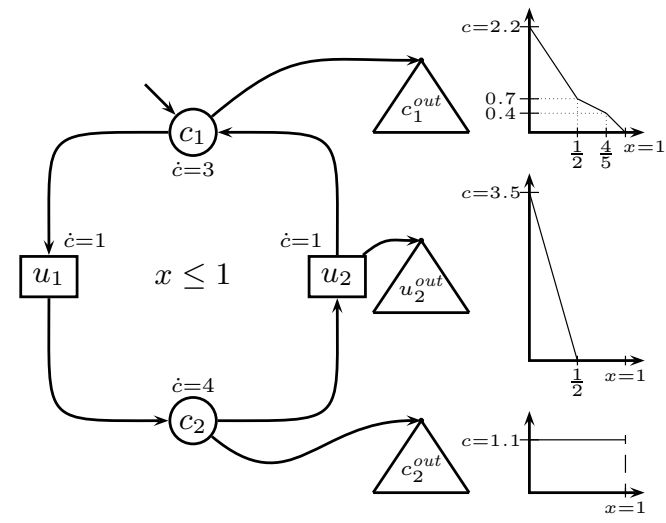

Fig. 1. Sample PTGA with outside cost functions.

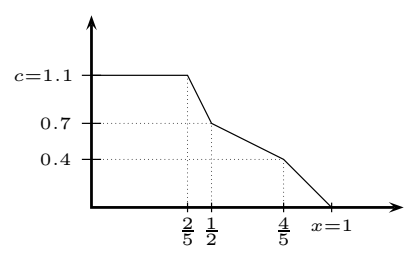

$\sigma\left(c_{2}, x\right)= \begin{cases}c_{2}^{\text {out }} & \text { if } 0 \leq x<2 / 5 \\ c_{2} & \text { if } 2 / 5 \leq x<1 / 2 \\ u_{2} & \text { if } 1 / 2 \leq x \leq 1\end{cases}$

Fig. 2. An optimal strategy in $c_{2}$, and the associated cost function.

In [12] the problem of computing cost-optimal winning strategies has been studied and shown computable for acyclic priced timed games. Furthermore, in [11] it is proven that computing optimal winning strategies for one-clock PTGA with stopwatch cost (i.e. cost are either zero or one) is decidable. [2] and [10] provide partial solutions to the general case of non-acyclic games: under the assumption of certain non-Zenoness behaviour of the underlying priced timed automata it is shown that it suffices only to consider strategies guaranteed to win within some given number $k$ of steps, or alternatively to unfold the given game $k$ times and reduce the problem to solving an acyclic game. To see how restricted these results are, it may be observed that the priced timed game in Fig. 1 does not belong to any of the above classes. In fact, in [11] it has recently been shown that the problem of determining cost-optimal winning strategies for priced timed games is not computable. Most recently, it has been shown that this negative result holds even for priced timed (game) automata with no more than three clocks [7].

In this paper we completely solve the computation of cost-optimal winning strategies for arbitrary priced timed (game) automata with one clock: we offer an algorithm for computing optimal costs, explain why optimal strategies need not always exist, whereas memoryless $\varepsilon$-optimal strategies exist and can be computed. 


\section{Definitions}

We write $x$ for the (unique) clock variable, and $\mathcal{X}=\{x\}$. A clock constraint for clock $x$ is an expression of the form $x \in I$ where $I$ is an interval over the reals with integer (or infinite) bounds which can have strict or non-strict bounds. As a shortcut, we may use expressions like $x \geq 5$ instead of $x \in[5,+\infty[$. The set of all clock constraints is denoted $\mathcal{B}(\mathcal{X})$. That a valuation $v: \mathcal{X} \rightarrow \mathbb{R}_{+}$satisfies a clock constraint $g$ is defined in a natural way ( $v$ satisfies $x \in I$ whenever $v(x) \in I$ ), and we then write $v \models g$. We denote by $v_{0}$ the valuation that assigns zero to clock $x$, by $v+t\left(t \in \mathbb{R}_{+}\right)$the valuation that assigns $v(x)+t$ to $x \in \mathcal{X}$.

A cost function is a piecewise affine function $f: \mathbb{R}_{+} \rightarrow \mathbb{R}_{+} \cup\{+\infty\}$ with negative slopes. We also require that if $\{+\infty\} \in f((n, n+1))$ for some integer $n$, then $f((n, n+1))=\{+\infty\}$, and that $f$ is continuous over all intervals $(n, n+1)$. We write CF for the set of all cost functions.

We define an extended notion of priced timed games, with outside cost functions and urgent locations. Those extra features will be needed throughout the proof. A 1-clock priced timed game with outside cost functions ( $\mathrm{PTG}_{f}$ for short) is a tuple $G=\left(Q_{c}, Q_{u}, Q_{f}, Q_{\mathrm{urg}}, Q_{\text {init }}, f_{\text {goal }}, T, \eta, P\right)$ where

- $Q_{c}$ is a finite set of controllable locations, $Q_{u}$ is a finite set of uncontrollable locations. Those sets are disjoint, and we define $Q=Q_{c} \cup Q_{u}$;

$-Q_{f}$ is the set of final locations (it is disjoint from $Q$ ).

- $Q_{\text {urg }} \subseteq Q_{u}$ indicates urgent uncontrollable locations;

- $Q_{\text {init }} \subseteq Q$ is the set of initial locations;

$-f_{\text {goal }}: Q_{f} \rightarrow \mathrm{CF}$ assigns to each final location a cost function;

$-T \subseteq Q \times \mathcal{B}(\mathcal{X}) \times 2^{\mathcal{X}} \times\left(Q \cup Q_{f}\right)$ is the set of transitions;

- $\eta: Q \rightarrow \mathcal{B}(\mathcal{X})$ defines the invariants of each location;

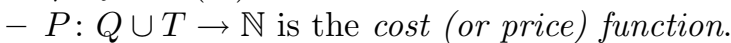

Standard (1-clock) priced timed games $[2,10]$ are $\mathrm{PTG}_{f}$ with $Q_{\mathrm{urg}}=\varnothing$ and, for any $q \in Q_{f}, f_{\text {goal }}(q)\left(\mathbb{R}_{+}\right)=\{0\}$ or $\{+\infty\}$.

In the following, $G$ will always refer to a $\mathrm{PTG}_{f}$, and we will not always rewrite the corresponding tuple. Similarly, $G^{\prime}$ will denote a $\mathrm{PTG}_{f}$ whose components are "primed".

We assume (w.l.o.g., see [6]) that the clock is bounded, i.e., there exists an integer $M$ such that for every location $q \in Q, \eta(q) \Rightarrow x \leq M$.

Let $G$ be a $\mathrm{PTG}_{f}$. The semantics of $G$ is given as a labeled timed transition system $\mathcal{T}=\left(S, S_{\text {init }}, \rightarrow\right)$ where $S \subseteq\left(Q \cup Q_{f}\right) \times \mathbb{R}_{+}$is the set of states ${ }^{3}, S_{\text {init }}=$ $Q_{\text {init }} \times\left\{v_{0}\right\}$ is the set of initial states, and the transitions relation $\rightarrow \subseteq S \times \mathbb{R}_{+} \times S$ is defined as:

1. (discrete transition) $(q, v) \stackrel{c}{\rightarrow}\left(q^{\prime}, v^{\prime}\right)$ if $q \notin Q_{f}$ and there exists $\left(q, g, R, q^{\prime}\right) \in$ $T$ such that $v(x) \models g, v^{\prime}=[R \leftarrow 0] v, v^{\prime}(x) \models \eta\left(q^{\prime}\right)$, and $c=P\left(q, g, R, q^{\prime}\right)$;

2. (delay transition) $(q, v) \stackrel{c}{\rightarrow}(q, v+t)$ if $q \notin Q_{\mathrm{urg}} \cup Q_{f}$, and $\forall 0 \leq t^{\prime} \leq t$, $v+t^{\prime} \models \eta(q)$, and $c=t \cdot P(q)$.

${ }^{3}$ Formally, $S \subseteq\left(Q \cup Q_{f}\right) \times\left(\mathbb{R}_{+}\right)^{\mathcal{X}}$, but we identify $v$ with $v(x)$ here. 
A run of $G$ is a (finite) path in the underlying transition system. Given $T, U \subseteq S$, we write $\operatorname{Run}_{G}(T, U)$ for the set of runs of $G$ issued from $t \in T$ and ending in $u \in U$. Given a run $\varrho$ and a position $v \in \varrho$ along that run, the prefix of $\varrho$ ending in $v$ is denoted by $\varrho_{\mid v}$. A run is maximal if either it is infinite, or no discrete transition is possible (even after a delay transition). A maximal run is accepting if it is finite and ends in a final location. Let $\varrho=s_{0} \stackrel{c_{0}}{\longrightarrow} s_{1} \stackrel{c_{1}}{\longrightarrow} \cdots \stackrel{c_{n-1}}{\longrightarrow} s_{n}$ be a run. Its cost, denoted $\operatorname{cost}(\varrho)$, is either $\sum_{i=0}^{n-1} c_{i}$ if $\varrho$ is not accepting, or $\sum_{i=0}^{n-1} c_{i}+f_{\text {goal }}\left(q_{n}\right)\left(v_{n}(x)\right)$, where $\left(q_{n}, v_{n}\right)=s_{n}$ if $\varrho$ is accepting. An accepting run is winning if it has finite cost.

Example. Reconsider the example depicted in Fig. 1. Here, a sample winning run is $\varrho=\left(c_{1}, 0\right) \stackrel{0}{\rightarrow}\left(u_{1}, 0\right) \stackrel{0.4}{\longrightarrow}\left(u_{1}, 0.4\right) \stackrel{0}{\rightarrow}\left(c_{2}, 0.4\right) \stackrel{0.4}{\longrightarrow}\left(c_{2}, 0.5\right) \stackrel{0}{\rightarrow}\left(c_{2}^{\text {out }}, 0.5\right)$ which has $\operatorname{cost} \operatorname{cost}(\varrho)=0.4 \times 1+0.1 \times 4+f_{\text {goal }}\left(c_{2}^{\text {out }}\right)(0.5)=1.9$.

A strategy is then a function $\sigma: \operatorname{Run}_{G}\left(Q \times \mathbb{R}_{+}, Q_{c} \times \mathbb{R}_{+}\right) \rightarrow\{\lambda\} \cup Q \cup Q_{f}$. Informally, a strategy tells in all controllable locations, what has to be done, and the special symbol $\lambda$ indicates to delay. A strategy $\sigma$ is memoryless if $\sigma(\varrho)=\sigma\left(\varrho^{\prime}\right)$ as soon as $\varrho$ and $\varrho^{\prime}$ end in the same state.

Let $\sigma$ be a strategy in $G$, and $\varrho_{0}$ a run in $G$ ending in $\left(q_{0}, x_{0}\right)$. A run $\varrho=$ $\left(q_{0}, x_{0}\right) \stackrel{c_{0}}{\longrightarrow}\left(q_{1}, x_{1}\right) \stackrel{c_{1}}{\longrightarrow} \cdots \stackrel{c_{n-1}}{\longrightarrow}\left(q_{n}, x_{n}\right)$ is a $\left(\sigma, \varrho_{0}\right)$-run if for all delay- (or discrete-) transitions $\left(q_{i}, x_{i}\right) \stackrel{c_{i}}{\longrightarrow}\left(q_{i+1}, x_{i+1}\right)$ where $q_{i} \in Q_{c}$, we have

- $\forall x \in\left[x_{i}, x_{i+1}\left[, \sigma\left(\varrho_{0} \cdot \varrho_{\mid x}\right)=\lambda\right.\right.$,

- $\sigma\left(\varrho_{0} \cdot \varrho_{\mid x_{i}}\right)=q_{i+1}$.

where $\varrho_{0} \cdot \varrho$ denotes the (usual) concatenation. In that case, we say that $\varrho$ is compatible with $\sigma$ after $\varrho_{0}$ (or that it is an outcome of $\sigma$ after $\varrho_{0}$ ). We write $\operatorname{Run}_{G, \sigma}\left(\varrho_{0}, U\right)$ for the set of such runs ending in $U$.

A strategy $\sigma$ is said accepting after (run) $\varrho_{0}$ whenever all maximal runs in $\operatorname{Run}_{G, \sigma}\left(\varrho_{0}\right)$ are accepting. If a strategy is not accepting from $\varrho_{0}$, we set its cost in $G$ after $\varrho_{0}, \operatorname{Cost}_{G}\left(\sigma, \varrho_{0}\right)$, to $+\infty$. Otherwise its cost in $G$ after $\varrho_{0}$ is given as: $\operatorname{Cost}_{G}\left(\sigma, \varrho_{0}\right)=\sup \left\{\operatorname{cost}(\varrho) \mid \varrho \in \operatorname{Run}_{G, \sigma}\left(\varrho_{0}, Q_{f} \times \mathbb{R}_{+}\right)\right\}$. Obviously, for any two runs $\varrho_{0}$ and $\varrho_{1}$ ending in $(q, x)$, the sets $\left\{\operatorname{Cost}_{G}\left(\sigma, \varrho_{0}\right) \mid \sigma\right.$ strategy in $\left.G\right\}$ and $\left\{\operatorname{Cost}_{G}\left(\sigma, \varrho_{1}\right) \mid \sigma\right.$ strategy in $\left.G\right\}$ are equal. An accepting strategy $\sigma$ after $\varrho_{0}$ is winning if $\operatorname{Cost}_{G}\left(\sigma, \varrho_{0}\right)$ is finite. We define for every state $s$ of $G$, the optimal cost of winning from $s$ as $\inf \left\{\operatorname{Cost}_{G}\left(\sigma, \varrho_{0}\right) \mid \sigma\right.$ strategy in $\left.G\right\}$ for some run $\varrho_{0}$ ending in $s$. We denote it $\operatorname{OptCost}_{G}(s)$. If $\operatorname{OptCost}_{G}(s)<+\infty$, the state $s$ is said winning in $G$. In that case, for every $\varepsilon>0$, for every run $\varrho_{0}$ ending in $s$, there exists a winning strategy $\sigma$ s.t. $\operatorname{OptCost}_{G}(s) \leq \operatorname{Cost}_{G}\left(\sigma, \varrho_{0}\right)<\operatorname{OptCost}_{G}(s)+\varepsilon$, and we say that $\sigma$ is $\varepsilon$-optimal from $\varrho_{0}$. A strategy $\sigma$ is optimal from $\varrho_{0}$ if $\operatorname{Cost}_{G}\left(\sigma, \varrho_{0}\right)=\operatorname{OptCost}_{G}(s)$ where $\varrho_{0}$ ends in state $s$.

A strategy $\sigma$ in $G$ is $(\varepsilon, N)$-acceptable (with $\varepsilon>0$, and $N \in \mathbb{N}$ ) whenever: (1) it is memoryless, (2) it is $\varepsilon$-optimal, (3) there exist $N$ (consecutive) intervals $\left(I_{i}\right)_{1 \leq i \leq N}$ partitioning $[0,1]$ such that for every location $q$, for every $1 \leq i \leq N$, for every integer $\alpha<M$, the function $x \mapsto \operatorname{Cost}_{G}(\sigma,(q, x))$ is affine on every interval $\alpha+I_{i}$, and the function $x \mapsto \sigma(q, x)$ is constant on $\alpha+I_{i}$.

\footnotetext{
${ }^{4}$ In the sequel, we might omit the subscripts $G$ when they are clear from the context.
} 


\section{Main result}

The main result of this paper is that optimal cost is computable and that almostoptimal memoryless strategies always exist and can be effectively computed. This is summarized by the following theorem:

Theorem 1. Let $G$ be a $P T G_{f}$. Then for every location $q$ in $G$, the function $x \mapsto \operatorname{OptCost}_{G}((q, x))$ is computable and piecewise-affine. Moreover, for every $\varepsilon>0$, there exists (and we can effectively compute) a strategy $\sigma$ in $G$ such that $\sigma$ is memoryless and $\varepsilon$-optimal in every state.

We will even prove a stronger result, which is that there exists $N \in \mathbb{N}$ such that for every $\varepsilon>0$, we can effectively compute an $(\varepsilon, N)$-acceptable strategy $\sigma$. The rest of this paper is devoted to a proof of this result.

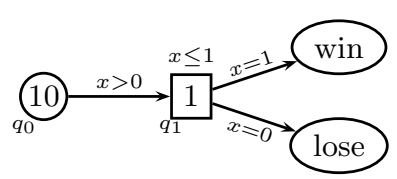

Fig. 3. A game with no optimal strategy

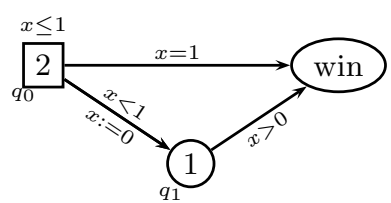

Fig. 4. A game where optimal strategies require memory

There are $\mathrm{PTG}_{f}$ for which no optimal strategies exist, as exemplified by Fig. 3: from $q_{0}$, the optimal cost is 1 , but a winning strategy consists in delaying in $q_{0}$ for some duration $\delta>0$, yielding a cost of $1+9 \delta$. This is why we compute, in the general case, $\varepsilon$-optimal strategies. In the same way, as witnessed by Fig 4 , it might be the case that optimal strategies exist but require some amount of memory: in the example of Fig 4, state $\left(q_{0}, x=0\right)$ is winning with optimal cost 2 , but no memoryless strategy can achieve that cost for sure.

\section{Simplifying Transformations}

In this section, we first explain how to restrict to simpler games while preserving the same optimal costs, and we then show how we can inductively compute optimal cost on those simpler games. We also explain how to compute almostoptimal strategies for those simpler games, and how to "lift" those strategies to the original game.

Our transformations consist in two steps: $(i)$ we restrict to $\mathrm{PTG}_{f}$ where the clock is bounded by 1 (denoted [0,1]-PTG ${ }_{f}$ ) (Section 4 ); (ii) we restrict to a $[0,1]-\mathrm{PTG}_{f}$ without resetting transition (Section 4). For each transformation, we prove that:

- the optimal cost in each state of the original game is identical to the optimal cost in some corresponding state in the transformed game, 
- we can derive an $\varepsilon$-optimal strategy in the original game from some $\varepsilon^{\prime}$ optimal strategy in the transformed game.

Section 5 is then devoted to computing the optimal cost and an almost-optimal strategy in the simpler game. For the sake of simplicity, we assume here that there are no discrete costs on transitions. A slight adaptation of the transformation for removing resets can be given for handling discrete costs as well.

Restricting to a $\mathbf{P T G}$ f bounded by $\mathbf{1}$. The idea of this construction is to reset the clock each time it reaches 1 , and to record in the discrete structure what should be the real integer part of the value of the clock (the clock will only store the fractional part of its real value).

Let $G$ be a $\mathrm{PTG}_{f}$. We build another $\mathrm{PTG}_{f} G^{\prime}$ such that for every $q^{\prime} \in Q^{\prime}$, $\eta^{\prime}\left(q^{\prime}\right)$ implies $0 \leq x \leq 1$, and $G^{\prime}$ is correct for computing optimal cost, in a sense which will be made clear later.

As we have assumed that $\mathrm{PTG}_{f}$ are bounded, we set $M$ the constant bound$\operatorname{ing} G$, and we define:

$$
\left\{\begin{array}{l}
Q_{x}^{\prime}=\left\{q_{[\alpha, \alpha+1]} \mid q \in Q_{x} \text { and } 0 \leq \alpha<M\right\} \text { for every } x \in\{c, u, f, \operatorname{urg}\} \\
Q_{\text {init }}^{\prime}=\left\{q_{[0,1]} \mid q \in Q_{\text {init }}\right\}
\end{array}\right.
$$

The set of transitions $T^{\prime}$ is composed of the following transitions (if $g$ is a guard, $g-\alpha$ denotes the same guard translated by $-\alpha$ ):

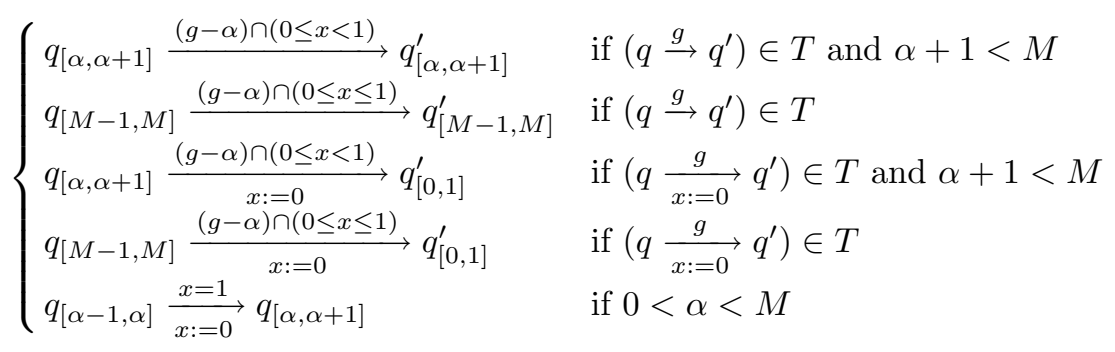

The invariant $\eta^{\prime}$ is defined by $\eta^{\prime}\left(q_{[\alpha, \alpha+1]}\right)=(0 \leq x \leq 1) \wedge(\eta(q)-\alpha)$ if $q \in Q$. The cost function $P^{\prime}$ is defined by $P^{\prime}\left(q_{[\alpha, \alpha+1]}\right)=P(q)$. The function $f_{\text {goal }}^{\prime}$ is defined by $f_{\text {goal }}^{\prime}\left(q_{[\alpha, \alpha+1]}\right)(x)=f_{\text {goal }}(q)(x+\alpha)$ for every $0 \leq x \leq 1$.

Note that all guards and invariants of $G^{\prime}$ are included in $[0,1]$, we say that $G^{\prime}$ is a $[0,1]-P T G_{f}$.

We define $f$ the function which maps every state $(q, x)$ of $G$ onto the state $\left(q_{[\alpha, \alpha+1]}, x-\alpha\right)$ of $G^{\prime}$ such that $0 \leq x-\alpha \leq 1$ and $x<M$ integer implies $x=\alpha$. We now state the following correctness result.

Proposition 2. For every state $(q, x)$ in $G, \operatorname{OptCost}_{G}(q, x)=\operatorname{OptCost}_{G^{\prime}}(f(q, x))$. Moreover, for every $\varepsilon>0$ and $N \in \mathbb{N}$, given an $(\varepsilon, N)$-acceptable strategy in $G^{\prime}$, we can compute an $(\varepsilon, N)$-acceptable strategy in $G$, and vice-versa. 
Removing resetting transitions from SCCs. We have restricted to games with a single clock. A strong property of this model is that each time a resetting transition is taken, then the very same state is visited (because the valuation is each time $v_{0}$ ). The construction for removing resetting transitions takes advantage of this property.

Let $G$ be a $\mathrm{PTG}_{f}$ with $n$ resetting transitions. From the previous reduction, we may assume that all the invariants and guards in $G$ imply that $0 \leq x \leq 1$. We build a $\mathrm{PTG}_{f} G^{\prime}$, made of $n+1$ copies of $G$, such that no strongly connected component (SCC for short) of $G^{\prime}$ contains a resetting transition.

We thus define $Q_{c}^{\prime}=Q_{c} \times\{0, \ldots, n\}, Q_{u}^{\prime}=Q_{u} \times\{0, \ldots, n\}$, and $Q_{f}^{\prime}=\left(Q_{f} \times\right.$ $\{0, \ldots, n\}) \cup\{r\}$. A location $(q, i) \in Q_{u}^{\prime}$ is urgent iff $q \in Q_{\text {urg. }}$. We let $Q_{\text {init }}^{\prime}=$ $Q_{\text {init }} \times\{0\}$. The outside cost functions are given by $f_{\text {goal }}^{\prime}((q, i))=f_{\text {goal }}(q)$, and $f_{\text {goal }}(r)=+\infty$. The invariant is given by $\eta^{\prime}((q, i))=\eta(q)$ for $q \in Q$. Transitions are defined as follows:

$$
\begin{cases}\left((q, i) \stackrel{g}{\rightarrow}\left(q^{\prime}, i\right)\right) \in T^{\prime} & \text { if }\left(q \stackrel{g}{\rightarrow} q^{\prime}\right) \in T \text { and } i \leq n \\ \left((q, i) \stackrel{g}{\rightarrow:=0}\left(q^{\prime}, i+1\right)\right) \in T^{\prime} & \text { if }\left(q \underset{g:=0}{\longrightarrow} q^{\prime}\right) \in T \text { and } i<n \\ ((q, n) \stackrel{g}{\rightarrow} r) \in T^{\prime} & \text { if }\left(q \underset{x:=0}{\longrightarrow} q^{\prime}\right) \in T \text { and } i=n\end{cases}
$$

Last, we set $P^{\prime}((q, i))=P^{\prime}(q)$ for every $q \in Q$, and the price of each transition of $T^{\prime}$ defined above is the price of the corresponding transition in $T$.

Proposition 3. For every state $(q, x)$ in the game $G$, OptCost ${ }_{G}((q, x))$ equals OptCost $_{G^{\prime}}(((q, 0), x))$. Moreover, for every $\varepsilon^{\prime}>0$ and $N^{\prime} \in \mathbb{N}$, given an $\left(\varepsilon^{\prime}, N^{\prime}\right)$ acceptable strategy in $G^{\prime}$, we can compute a $\left(2 \varepsilon^{\prime}, N^{\prime}\right)$-acceptable strategy in $G$.

We have thus reduced our problem to computing optimal cost and almostoptimal winning strategies in $G^{\prime}$. In $G^{\prime}$, this can be done by first computing it in the $n$th copy of $G$, and then in the $(n-1)$ th copy of $G$, etc.

\section{Computing almost-optimal strategies}

We have restricted our problem to $[0,1]-\mathrm{PTG}_{f}$ without resets. We can also easily restrict to such $\mathrm{PTG}_{f}$ containing only one SCC: if we can compute the optimal costs and an $(\varepsilon, N)$-acceptable strategy on an SCC, we will be able to handle the general case by working first on the deepest SCC, and then replace it by the corresponding outside function (and an $(\varepsilon, N)$-acceptable strategy).

Thus, we now assume that we only work on a $[0,1]-\mathrm{PTG}_{f}$ without resets and based on an SCC. We prove the following result, which will imply Theorem 1.

Theorem 4. Let $G$ be a $[0,1]-P T G_{f}$ without reset such that $\left(Q_{c} \cup Q_{u}, T\right)$ is an $S C C$ (or contains only one location). Then:

H1. $\operatorname{OptCost}_{G}(q, x)$ is computable for every $q \in Q$ and every $x \in[0,1]$;

H2. for every location $q \in Q, x \in[0,1] \mapsto \operatorname{OptCost}_{G}(q, x)$ is a cost function whose finitely many segments either have slope $-c$ where $c \in P(Q)$, or are fragments of the outside cost functions of $G$; 
H3. there exists an integer $N$ such that, for any $\varepsilon>0$, we can compute an $(\varepsilon, N)$-acceptable strategy in $G$ for every $q \in Q$ and every $x \in[0,1]$.

The rest of this section is devoted to the proof of this theorem, which is by induction on the number of non-urgent locations in $G$. First we prove the base case of the induction, that is when the game is only composed of urgent locations, or of a single controllable location.

- Proving properties H1 and H2 in the case where $G$ contains only one location is handled straightforwardly, by combining the outside cost functions of $G$ with the cost rate of the location. Property $\mathrm{H} 3$ requires more care. Let $q$ be a (controllable) location with a bunch of outside cost functions $\left\{f_{\text {goal }}\left(q^{\prime}\right) \mid\right.$ $\left.q^{\prime} \in Q_{f}\right\}$. Define the function $s: x \rightarrow \min \left\{f_{\text {goal }}\left(q^{\prime}, x\right) \mid q^{\prime} \in Q_{f}\right\}$. Then $\operatorname{OptCost}_{G}(q, x)=\inf _{x \leq x^{\prime} \leq 1} P(q) \cdot\left(x^{\prime}-x\right)+s\left(x^{\prime}\right)$. Let $\varepsilon>0$. We then define the strategy $\sigma$ as follows:

$$
\sigma(q, x)=\left\{\begin{array}{l}
q^{\prime} \text { if } \operatorname{OptCost}_{G}(q, x)=f_{\text {goal }}\left(q^{\prime}\right)(x) \\
\lambda \text { if } \operatorname{OptCost}_{G}(q, x)<s(x) \text { and either } s(1)<+\infty \\
\text { or } x \leq 1-\varepsilon /(2 P(q)) \\
q^{\prime} \text { if } \operatorname{OptCost}_{G}(q, x)<s(x), s(1)=+\infty, 1-\varepsilon /(2 P(q))<x<1, \\
\quad \text { and } \lim _{x \rightarrow 1^{-}} f_{\text {goal }}\left(q^{\prime}\right)(x)=\lim _{x \rightarrow 1^{-}} s(x)
\end{array}\right.
$$

It is not difficult to check that $\sigma$ is $(\varepsilon, N)$-acceptable for some $N$ which is independent of $\varepsilon$.

- The case where $G$ contains an SCC with only urgent (thus uncontrollable) locations is also straightforward, since the opponent can force the game to never reach a final location, and the optimal cost is then $+\infty$. If the game is composed of a single urgent location, then this is also easy.

We now assume that $G$ is an SCC composed of at least two locations, $n$ of which are non-urgent. We select one of the non-urgent locations having least cost, and denote it with $q_{\text {min }}$, and, depending on the nature (controllable or not) of $q_{\text {min }}$, we explain how we prove that Theorem 4 holds for $G$ if it holds for SCCs having at most $(n-1)$ non-urgent locations.

Case: $\boldsymbol{q}_{\min }$ is controllable. For handling this case, we will prove that the rough intuition that there is no need to delay twice in $q_{\min }$, but we better delay longer in $q_{\mathrm{min}}$ is indeed correct.

From the game $G$, we construct a game $G^{\prime}$, made of two copies of $G$, such that each SCC of the new game contains one location less (see Fig. 5). We define $Q_{c}^{\prime}=\left(Q_{c} \backslash\left\{q_{\min }\right\}\right) \times\{0,1\} \cup\left\{q_{\min }\right\}, Q_{u}^{\prime}=Q_{u} \times\{0,1\}, Q_{f}^{\prime}=Q_{f} \times\{0,1\} \cup\{r\}$, $Q_{\text {urg }}^{\prime}=Q_{\text {urg }} \times\{0,1\}, Q_{\text {init }}^{\prime}=Q_{\text {init }} \times\{0\}, f_{\text {goal }}^{\prime}((q, i))=f_{\text {goal }}(q)$ if $q \in Q_{f}$, and $f_{\text {goal }}^{\prime}(r)=+\infty, \eta^{\prime}((q, i))=\eta(q), \eta^{\prime}\left(q_{\min }\right)=\eta\left(q_{\min }\right), P^{\prime}((q, i))=P(q)$ for every $(q, i) \in Q_{c}^{\prime} \cup Q_{u}^{\prime}$. The set of transitions is

$$
\begin{aligned}
T^{\prime} & =\left\{(q, i) \stackrel{g, R}{\longrightarrow}\left(q^{\prime}, i\right) \mid q \stackrel{g, R}{\longrightarrow} q^{\prime}, \text { and } q, q^{\prime} \neq q_{\min }\right\} \\
& \cup\left\{(q, 0) \stackrel{g, R}{\longrightarrow} q_{\min },(q, 1) \stackrel{g, R}{\longrightarrow} r \mid\left(q \stackrel{g, R}{\longrightarrow} q_{\min }\right) \in T\right\} \\
& \cup\left\{q_{\min } \stackrel{g, R}{\longrightarrow}\left(q^{\prime}, 1\right) \mid\left(q_{\min } \stackrel{g, R}{\longrightarrow} q^{\prime}\right) \in T\right\} .
\end{aligned}
$$



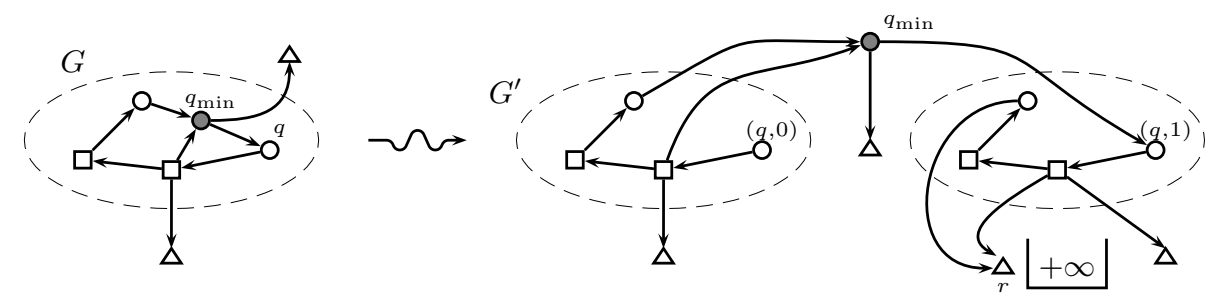

Fig. 5. Case $q_{\min }$ (in grey) controllable

We prove the following lemma, which establishes properties H1 and H2.

Lemma 5. For every $(q, x) \in\left(Q \backslash\left\{q_{\min }\right\}\right) \times[0,1]$, we have OptCost ${ }_{G}(q, x)=$ $\operatorname{OptCost}_{G^{\prime}}((q, 0), x)$. For every $x \in[0,1]$, OptCost ${ }_{G}\left(q_{\min }, x\right)=\operatorname{OptCost}_{G^{\prime}}\left(q_{\min }, x\right)$.

It remains to prove property $\mathrm{H} 3$. We fix the integer $N^{\prime}$ for $G^{\prime}$. We fix some $\varepsilon>0$, and take $\varepsilon^{\prime}=\frac{\varepsilon}{3}$. We take $\sigma^{\prime}$ an $\left(\varepsilon^{\prime}, N^{\prime}\right)$-acceptable strategy in $G^{\prime}$. We then define $\sigma$ as follows:

$$
\sigma(q, x)= \begin{cases}\sigma^{\prime}((q, 1), x) & \text { if } \operatorname{Cost}_{G^{\prime}}\left(\sigma^{\prime},((q, 1), x)\right) \leq \operatorname{OptCost}_{G^{\prime}}\left(q_{\min }, x\right) \\ \sigma^{\prime}((q, 0), x) & \text { otherwise }\end{cases}
$$

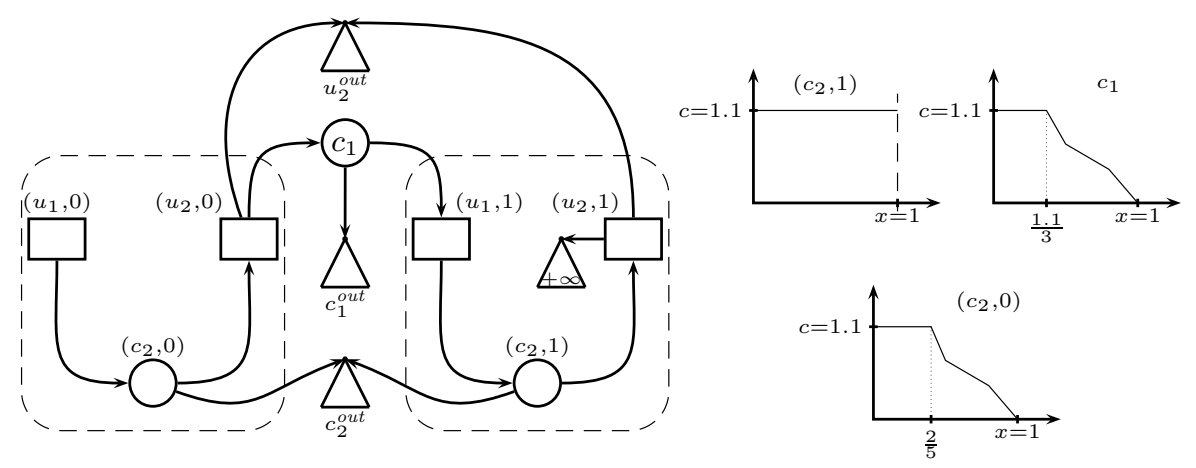

Fig. 6. Running example after unwinding.

Fig. 7. Optimal costs.

Example. Returning to the running example of Fig. 1 with $u_{1}$ and $u_{2}$ urgent, performing the above transformation with respect to $c_{1}$ gives the $\mathrm{PTG}_{f}$ depicted in Fig. 6. The optimal cost functions are depicted in Fig. 7 and the resulting winning strategy for $c_{2}$ is, according to (1), the strategy of $\left(c_{2}, 1\right)$ when $x \leq \frac{1.1}{3}$ and $\left(c_{2}, 0\right)$ otherwise.

Obviously, the strategy $\sigma$ is memoryless. We need to establish that the function $x \mapsto \operatorname{Cost}_{G}(\sigma,(q, x))$ consists of at most $N$ pieces, and that $\sigma$ is $\varepsilon$-optimal.

Proposition 6. Strategy $\sigma$ is winning and there exists a fixed (independant of $\varepsilon$ ) integer $N$ such that $\sigma$ is $(\varepsilon, N)$-acceptable. 


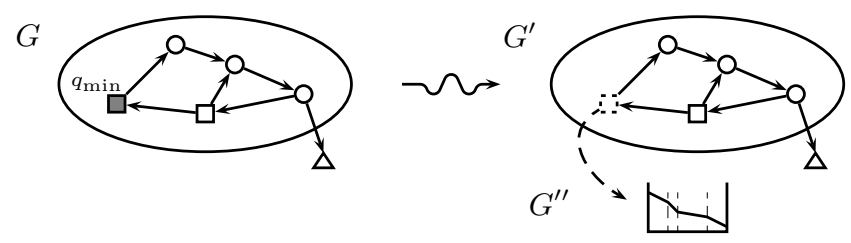

Fig. 8. When it is uncontrollable, $q_{\min }$ is made urgent (in dash line here).

Case: $\boldsymbol{q}_{\min }$ is uncontrollable. The intuition is that the opponent will prefer delays in other locations than $q_{\text {min }}$ whenever possible. We attempt to enforce this by a transformation of the game where location $q_{\min }$ is urgent, as depicted in Fig. 8. Formally, given a $[0,1]-\mathrm{PTG}_{f}$ without resets $G$, we define $G^{\prime}$ with $Q_{\text {urg }}^{\prime}=Q_{\text {urg }} \cup\left\{q_{\min }\right\}$ and $Q_{u}^{\prime}=Q_{u} \backslash\left\{q_{\min }\right\}$.

Obviously enough, since we restrict the possible moves for the opponent in $G^{\prime}$, we have for every state $(q, x)$, OptCost $G^{\prime}(q, x) \leq \operatorname{OptCost}_{G}(q, x)$.

However, the converse inequality is not correct over $[0,1]$, and we will need a more complex construction to handle this case. We now explain how to iteratively compute the optimal costs in $G$. Fig. 9 gives an overview of the computation described below.
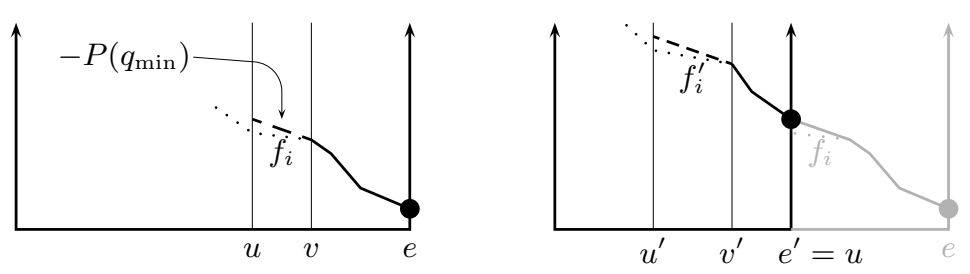

Fig. 9. Successive computations when $q_{\min }$ is uncontrollable

Clearly, we can compute OptCost ${ }_{G}\left(q_{\text {min }}, 1\right)$ (indeed, OptCost ${ }_{G}\left(q_{\text {min }}, 1\right)=$ OptCost $_{G^{\prime}}\left(q_{\mathrm{min}}, 1\right)$, since when $x=1$, time cannot elapse any more and the same moves are available in $G^{\prime}$ and in $G$ ). This initializes our iterative computation.

Now, assume we can compute $\operatorname{OptCost}_{G}\left(q_{\text {min }}, e\right)$ for some $e \in[0,1]$. We can apply the induction hypotheses $\mathrm{H} 1-\mathrm{H} 3$ to $G^{\prime}$. In particular, $f: x \in[0, e] \mapsto$ $\operatorname{OptCost}_{G^{\prime}}\left(q_{\text {min }}, x\right)$ is a cost function satisfying the requirements of item $\mathrm{H} 2$. Writing $f_{1}, \ldots, f_{n}$ for the successive affine functions constituting $f$, we pick the smallest index $i$ such that for every $j>i$, function $f_{j}$ has slope less than or equal to $-P\left(q_{\min }\right)$. If $i>0$, we note $[u, v]$ the domain of $f_{i}$ (see Fig. 9).

Lemma 7. If $i=0$, for all $(q, x) \in Q \times[0, e], \operatorname{OptCost}_{G}(q, x)=\operatorname{OptCost}_{G^{\prime}}(q, x)$. If $i>0$, for all $(q, x) \in Q \times[v, e]$, OptCost ${ }_{G}(q, x)=\operatorname{OptCost}_{G^{\prime}}(q, x)$.

We now explain how to compute $\operatorname{OptCost}_{G}\left(q_{\min }, x\right)$ for $x \in[u, v]$; we prove the following lemma: 
Lemma 8. If $i>0$, then for all $(q, x) \in Q \times[u, v]$, we have $\operatorname{OptCost}_{G}\left(q_{\min }, x\right)=$ $(v-x) P\left(q_{\min }\right)+f(v)$.

The optimal cost in states $(q, x)$ with $x \in[u, v]$ can then be computed by considering the $\mathrm{PTG}_{f} G^{\prime \prime}$, restricted to $x \in[u, v]$, and obtained from $G^{\prime}$ by making $q_{\text {min }}$ a goal location with cost function equal to $x \mapsto \operatorname{OptCost}_{G}\left(q_{\text {min }}, x\right)$, which is then viewed as an outside cost function, see Fig. 9.

We can then repeat the procedure above on the interval $[0, u]$ (i.e. by setting $e=u$ ): compute $f^{\prime}: x \mapsto \operatorname{OptCost}_{G^{\prime}}\left(q_{\text {min }}, x\right)$ with $x \in[0, u]$, select an interval $\left[u^{\prime}, v^{\prime}\right]$ where $f_{i}^{\prime}$ has slope larger than or equal to $-P\left(q_{\text {min }}\right)$, and so on, replace that part with an affine function with slope $-P\left(q_{\min }\right)$, and continue with the interval $\left[0, u^{\prime}\right]$. We now explain why this process terminates: since they have slopes strictly greater than $-P\left(q_{\min }\right), f_{i}$ and $f_{i}^{\prime}$ are fragments of outside cost functions, according to hypothesis H2. If they have different slopes, then they are obviously parts of two different fragments of outside cost functions. If they have the same slopes, then they are fragments of two different parts of outside cost functions, since they are joined by affine functions with slopes less than (or equal to $\left.-P\left(q_{\min }\right)\right)$. Since there are only finitely many affine functions constituting the outside cost functions, our procedure terminates.

At each step of the procedure above, we can also compute $(\varepsilon, N)$-acceptable strategies, and merge them.

\section{Conclusion}

In this paper we have proven that optimal cost for arbitrary priced timed games with one clock is a computable problem, and that $\varepsilon$-optimal memoryless strategies may effectively be obtained. The complexity of our procedure is quite high, running in 3-EXPTIME, while the best known lower bound for this problem is PTIME. Our future works of course include tightening these bounds.

As a consequence of our result it may be shown that the iterative semialgorithm proposed in [10] always terminates for priced timed games with one clock. Cost functions $\operatorname{cost}_{G}^{i}$ are inductively defined, which for any location $q \in Q$ and any clock value $v$, give the optimal cost of winning from the state $(q, v)$ within at most $i$ steps (we count the number of steps in a run $\rho$ by the number of delayand-action fractions). Now Theorem 4 ensures that we can find a fixed $N$ such that for any $\varepsilon>0$ we can compute an $(\varepsilon, N)$-acceptable strategy. In particular this guarantees that we can find $\varepsilon$-optimal strategies which are guaranteed to win within $N \cdot|Q|$ steps for any $\varepsilon>0$. Consequently, $\left\langle\operatorname{cost}_{G}^{i}\right\rangle_{i=1}^{\infty}$ (the semialgorithm of [10]) converges after at most $N \cdot|Q|$ iterations to the optimal cost of winning. A prototype implementation of this iterative algorithm is available at http://www.cs.aau.dk/ illum/tools/1ptga/.

As future work we would like to determine what happens with priced timed games using two clocks, but this seems really difficult as our approach heavily relies on the fact that there is only one clock. 


\section{References}

1. Y. Abdeddaïm, E. Asarin, and O. Maler. Scheduling with timed automata. 2006.

2. R. Alur, M. Bernadsky, and P. Madhusudan. Optimal reachability in weighted timed games. In Proc. 31st Intl Coll. Automata, Languages and Programming (ICALP'04), LNCS 3142, p. 122-133. Springer, 2004.

3. R. Alur, C. Courcoubetis, and Th. A. Henzinger. Computing accumulated delays in real-time systems. In Proc. 5th Intl Conf. Computer Aided Verification (CAV'93), LNCS 697, p. 181-193. Springer, 1993.

4. R. Alur and D. Dill. A theory of timed automata. Theor. Comp. Science, 126(2):183-235, 1994.

5. R. Alur, S. La Torre, and G. J. Pappas. Optimal paths in weighted timed automata. In Proc. 4th Intl Workshop Hybrid Systems: Computation and Control (HSCC'01), LNCS 2034, p. 49-62. Springer, 2001.

6. G. Behrmann, A. Fehnker, Th. Hune, K. G. Larsen, P. Pettersson, J. Romijn, and F. Vaandrager. Minimum-cost reachability for priced timed automata. In Proc. 4th Intl Workshop Hybrid Systems: Computation and Control (HSCC'01), LNCS 2034, p. 147-161. Springer, 2001.

7. P. Bouyer, Th. Brihaye, and N. Markey. Improved undecidability results on weighted timed automata. Inf. Proc. Letters, (5):188-194, June 2006.

8. P. Bouyer, E. Brinksma, and K. G. Larsen. Staying alive as cheaply as possible. In Proc. '7th Intl Workshop Hybrid Systems: Computation and Control (HSCC'04), LNCS 2993, p. 203-218. Springer, 2004.

9. P. Bouyer, E. Brinksma, and K. G. Larsen. Optimal infinite scheduling for multipriced timed automata. Form. Meth. in Syst. Design, 2006. To appear.

10. P. Bouyer, F. Cassez, E. Fleury, and K. G. Larsen. Optimal strategies in priced timed game automata. In Proc. 24th Conf. Foundations of Software Technology 86 Theoretical Computer Science (FSTETCS'04), LNCS 3328, p. 148-160. Springer, 2004.

11. Th. Brihaye, V. Bruyère, and J.-F. Raskin. On optimal timed strategies. In Proc. 3rd Intl Conf. Formal Modeling and Analysis of Timed Systems (FORMATS'05), LNCS 3821, p. 49-64. Springer, 2005.

12. S. La Torre, S. Mukhopadhyay, and A. Murano. Optimal-reachability and control for acyclic weighted timed automata. In Proc. 2nd IFIP Intl Conf. Theoretical Computer Science (IFIPTCS'02), IFIP Conf. Proc. 223, p. 485-497. Kluwer, 2002.

13. K. G. Larsen, G. Behrmann, E. Brinksma, A. Fehnker, Th. Hune, P. Pettersson, and J. Romijn. As cheap as possible: Efficient cost-optimal reachability for priced timed automata. In Proc. 13th Intl Conf. Computer Aided Verification (CAV'01), LNCS 2102, p. 493-505. Springer, 2001.

14. K. G. Larsen and J. I. Rassmussen. Optimal conditional reachability for multipriced timed automata. In Proc. 8th Intl Conf. Foundations of Software Science and Computation Structures (FoSSaCS'05), LNCS 3441, p. 234-249. Springer, 2005.

15. J. I. Rasmussen, K. G. Larsen, and K. Subramani. Resource-optimal scheduling using priced timed automata. In Proc. 10th Intl Conf. Tools and Algorithms for the Construction and Analysis of Systems (TACAS'04), LNCS 2988, p. 220-235. Springer, 2004.

16. UPPAAL CORA. http://www.cs.aau.dk/ behrmann/cora/, Jan. 2006. 